\section{Diurnal and nocturnal sleep stage patterns following sleep deprivation*}

\author{
RALPH J. BERGER, JAMES M. WALKER, THOMAS D. SCOTT \\ LINDA J. MAGNUSON and STEPHEN L. POLLACK
}

Division of Natural Sciences I, University of California, Santa Cruz, Calif. 95060

The extent to which the propensities for Stage 4 and REM sleep are dependent upon amount of sleep loss, length of prior wakefulness, and circadian factors was examined. Diurnal and nocturnal sleep stage patterns in eight male and four female Ss were studied following 24 and $36 \mathrm{~h}$ of sleep deprivation, respectively. Stage 4 increased on day $(p<.01)$ and night $(p<.001)$ recovery but remained confined to the first third of sleep on both conditions. REM latency was reduced on day recovery $(p<.05)$, but percentage REM did not change following sleep deprivation. The propensity for Stage 4 appears to be influenced by the amount of sleep loss and that for REM by circadian factors.

Rapid eye movement (REM) and Stage 4 sleep are differentially distributed within uninterrupted nocturnal sleep (Dement \& Kleitman, 1957a, b; Williams, Agnew, \& Webb, 1964,1966 ). Stage 4 predominates during the first third of the night, whereas REM increases progressively, reaching a plateau during the last third of the night, so that it remains constant even when sleep is extended for longer than usual (Phoebus, Taub, Globus, \& Drury, 1970; Verdone, 1968).

If naps are taken subsequent to a regular night of sleep, the relative distribution of REM and Stage 4 changes as the naps are taken progressively later in the day (Maron, Rechtschaffen, \& Wolpert, 1964; Webb \& Agnew, 1967; Webb, Agnew, \& Sternthal, 1966). Naps taken early in the morning resemble a continuation of the terminal portion of the previous night's sleep, with large amounts of REM and negligible amounts of Stage 4 . Naps taken in the evening resemble the first portion of night sleep, with large amounts of Stage 4 and small amounts of REM. Naps taken midday or in the early afternoon have an intermediate pattern.

Are the propensities for REM and Stage 4 sleep influenced by the length of time a person has been awake since his major sleep period, by how much of his normal sleep he might have lost, by preexisting circadian rhythms, or by a combination of these three factors? Earlier work indicates that amounts of Stage 4 are dependent upon one or both of the first two factors, since Stage 4 is increased on

* The assistance of Joseph Avellar, Cecil Burchfiel, Anita Harten, James Rossie, Willard Snow, Marcum Sproule, Christine Summers, Susan Thatcher, Thomas Tower, and Fredric Wiebe is gratefully acknowledged. Supported in part by National Science Foundation Grant GB-8782. deprivation (Berger \& Oswald, 1962; Kales, Tan, Kollar, Naitoh, Preston, \& Malmstrom, 1970; Webb \& Agnew, 1965; Williams, Hammack, Daly, Dement, \& Lubin, 1964). This study was designed to differentiate between the relative influences of these three factors by examining diurnal and nocturnal sleep-stage patterns on recovery from 24 and $36 \mathrm{~h}$ of sleep deprivation, respectively. METHOD

The Ss were eight male and four female college students who had been instructed to maintain their regular sleep habits and to have abstained from drugs, including alcohol.

The Ss were studied in pairs, sleeping in separate bedrooms, on five different occasions, separated by at least 1 week. On three nights, Ss retired at 12:00 a.m.; the first two of nights following partial or total sleep these served as adaptation nights, and the third was used as a baseline of their normal nocturnal sleep patterns. On two other occasions, Ss stayed awake for an entire night while monitoring sleep recordings in the laboratory as part of an upper division psychology course requirement. On one of these occasions they went to bed at 9:00 a.m. and on the other, the following night, at 12:00 a.m., thereby undergoing 24 and $36 \mathrm{~h}$ of sleep deprivation, respectively. The order of the two sleep-deprivation conditions was counterbalanced among Ss.

Electrodes were placed for recording electroencephalograms, chin e l e c trom yograms, and electrooculograms, and the records were coded and scored blind for sleep stages, by 30-sec epochs, according to standard procedures (Rechtschaffen \& Kales, 1968). On all occasions, recordings were taken for a period of $8 \mathrm{~h}$ following which Ss were awakened.

Comparisons between sleep conditions were made by one-way analysis of variance for repeated measurements (Winer, 1962). Duncan's multiple range test was used to compare specific means. Hourly distribution of sleep stages and $w$ akefulness were compared with $t$ tests for correlated means.

\section{RESULTS}

Patterns of sleep on recovery following both sleep-deprivation conditions were similar in many respects but differed from baseline sleep. In other respects, the recovery conditions were different from each other, as well as from baseline sleep.

Table 1

Minutes of Total Sleep Time, Initial Wakefulnes, REM Latency, and Lensth of REM Cycle, and Number of RBM Periods

\begin{tabular}{|c|c|c|c|c|c|c|}
\hline & \multicolumn{2}{|c|}{ Baseline } & \multicolumn{2}{|c|}{ Day Recovery } & \multicolumn{2}{|c|}{ Night Recovery } \\
\hline & Mean & SD & Mean & SD & Mean & SD \\
\hline $\begin{array}{l}\text { Total Sleep Time } \\
\text { Initial Wakefuiness } \\
\text { REM Latency } \\
\text { REM Cycle Length } \\
\text { REM Periods }\end{array}$ & $\begin{array}{c}431.7 * \\
17.4 \dagger \\
110.4 \\
101.5 \\
4.08+\end{array}$ & $\begin{array}{l}46.4 \\
11.9 \\
50.0 \\
19.8 \\
1.00\end{array}$ & $\begin{array}{l}427.8 * * \\
7.2 \\
70.0+t \\
87.2 \\
4.92\end{array}$ & $\begin{array}{c}32.0 \\
6.5 \\
31.5 \\
14.0 \\
0.90\end{array}$ & $\begin{array}{r}468.5 \\
9.9 \\
105.1 \\
88.2 \\
4.75\end{array}$ & $\begin{array}{c}8.1 \\
13.0 \\
45.1 \\
12.6 \\
0.62\end{array}$ \\
\hline
\end{tabular}

$t_{p}<.05-$ Significantly different from day and night recovery.

$++p<.05-$ Significantly different from baseline and night recovery.

${ }^{*} p<.05, *^{*} p<.01-$ Significantly different from night recovery.

Table 2

Percentage of Total Sleep Time Spent in Each Sleep Stage

\begin{tabular}{|c|c|c|c|c|c|c|}
\hline \multirow[b]{2}{*}{ Sleep Stage } & \multicolumn{2}{|c|}{ Baseline } & \multicolumn{2}{|c|}{ Day Recovery } & \multicolumn{2}{|c|}{ Night Recovery } \\
\hline & Mean & SD & Mean & SD & Mean & SD \\
\hline 1 & 13.0 & 4.5 & 13.4 & 4.0 & $7.8+$ & 5.0 \\
\hline 2 & 55.0 & 7.2 & 53.0 & 6.2 & 55.7 & 5.9 \\
\hline 3 & 10.0 & 3.7 & 10.7 & 3.4 & 13.3 & 5.5 \\
\hline 4 & 2.5 & 3.3 & $5.1^{*}$ & 6.8 & $5.8 * *$ & 6.1 \\
\hline REM & 19.5 & 4.7 & 17.8 & 5.0 & 17.4 & 4.8 \\
\hline
\end{tabular}

$* p<.01, * * p<.001-$ Significantly different from baseline.

tp $<.01-$ Significantly different from baseline and day recovery. 


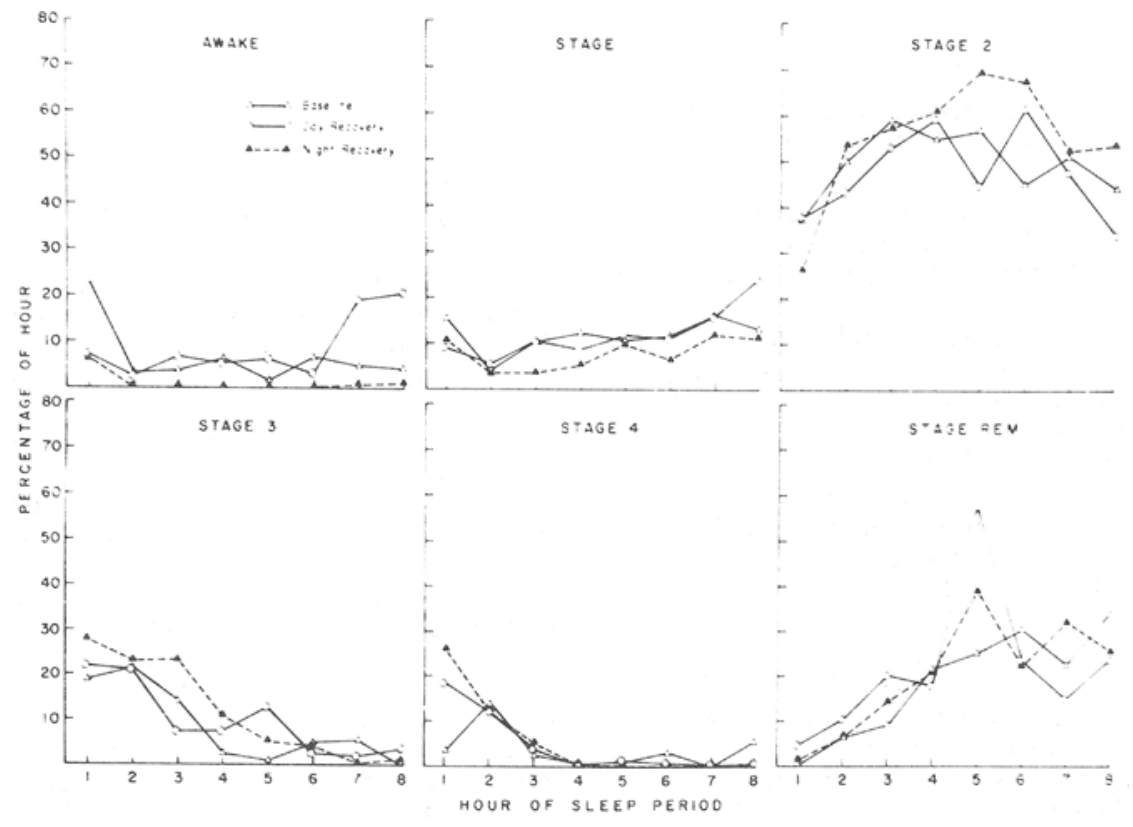

Fig. 1. Percentage of each successive hour spent awake and in each sleep stage under each condition.

\section{Similarities Between}

Recovery Conditions

The Ss fell asleep more rapidly, with less initial wakefulness $(p<.05)$ prior to first entering Stage 2, on both recovery conditions (Table 1 ).

Percentage of Stage 4 was higher on both day recovery $(p<.01)$ and night recovery $(p<.001)$ than on baseline nights (Table 2). This was especially true for the first hour of sleep $(p<.001$, Fig. 1) and reflected quicker entry into Stage 4 as well as greater amounts of Stage 4

The total number of REM periods was greater on both recovery conditions than on baseline nights $(p<.05)$ and reflected decreases in the length of the REM cycle which just failed to reach statistical significance $(p<.10$, Table 1$)$.

\section{Differences Between}

Recovery Conditions

Total time spent asleep was greater on night recovery than on baseline nights $(p<.05)$ and day recovery ( $p<.01$, Table 1). Sleep on night recovery was sounder, with less Stage 1 ( $p<.01$, Table 2) and fewer transitions to Stage 1 from other sleep stages $(\bar{x}=24.8, p<.05)$, than on baseline nights $(\bar{x}=35.0)$ and day recovery $(\bar{x}=39.6)$. Ss tended to wake up earlier on day recovery than on night recovery so that wakefulness was increased in the seventh $(p<.05)$ and eighth $(p<.05)$ hours of the recording session (Fig. 1).

Latency to the first REM period was shorter on day recovery than on baseline nights or night recovery
( $p<.05$, Table 1 ), but percentage REM did not change following either sleep-deprivation condition (Table 2 ).

\section{DISCUSSION}

The increase in Stage 4 following both sleep-deprivation conditions confirms earlier findings (Berger \& Oswald, 1962; Kales et, al, 1970; Williams et al, 1964). Ss lost one night's sleep on both sleep-deprivation conditions, but amounts of Stage 4 did not differ between the two conditions, even though Ss had been awake for $36 \mathrm{~h}$ on the night recovery condition as compared with $24 \mathrm{~h}$ on the day recovery condition. It, therefore, appears that the propensity for Stage 4 is primarily determined by the amount of habitual sleep a person has lost rather than the total time he has remained awake. The distribution of Stage 4 also remained independent of the normal circadian rhythm, being largely confined to the first third of sleep under all conditions.

By contrast, amounts of REM sleep did not differ among the three conditions. In an earlier study, REM was reduced during nocturnal sleep after $108 \mathrm{~h}$ of total sleep deprivation (Berger \& Oswald, 1962). The absence of a similar reduction in the present study may be due to the shorter length of sleep deprivation, although extreme lengths of deprivation of up to $205 \mathrm{~h}$ have been reported to lead to increases in REM sleep (Kales et al, 1970). Nevertheless, the distribution of REM sleep differed between the conditions. The shortened REM latency on day recovery indicates a persisting influence of the normal circadian distribution of REM sleep, in spite of sleep deprivation. However, the amounts of REM sleep in the first few hours of day recovery sleep were no higher than in the other two conditions, which suggests that Stage 4 sleep takes priority on recovery from sleep deprivation independently of preexisting circadian influences.

Weitzman, Kripke, Goldmacher, McGregor, \& Nogeire (1970) recently described sleep patterns following acute reversal of the sleep-waking cycle after 1 night without sleep. Although their data on daytime sleep was computed over 3-day periods rather than for each individual sleep period, their results are generally similar to these. After reversal, diurnal sleep was characterized by shorter REM latencies, shorter episodes of Stage 2, and more wakefulness in the last third of sleep than prior to reversal. Stage 4 predominated in the first third of the sleep period before and after reversal. On the other hand, we did not confirm their findings of an increased number of stage changes, decreased duration of REM periods, or increased number of interruptions of REM periods during daytime sleep. Using the same criteria for defining a REM period, as successive episodes of REM separated by less than $15 \mathrm{~min}$, no differences were found in the number of interrupted REM periods, total number of interruptions, or in the proportion of total number of REM periods which were interrupted between the three conditions. Possibly, the changes Weitzman et al described were not present on the first reversal day of sleep in their study but developed only on the second or third day, so that their 3-day block of data is not strictly comparable with the day recovery condition of this study.

\section{REFERENCES}

BERGER, R. J., \& OSWALD, I. Effects of sleep deprivation on behaviour. subsequent sleep, and dreaming. Journal of Mental Science, 1962,108, 457-465.

DEMENT, W., \& KLEITMAN, N. Cyclic variations in EEG during sleep and their relation to eye movements. body motility, and dreaming. Electroencephalography \& Clinical Neurophysiology, 1957a, 9, 673-690.

DEMENT, W. \& KLEITMAN, N. The relation of eye movements during sleep to dream activity: An objective method for the study of dreams. Joumal of Experimental Psychology, 1957b, 53, 339-346.

KALES, A., TAN, T. L., KOLLAR, E. J.. NAITOH, P. PRESTON, T. A.. \& MALMSTROM, E. J. Sleep patterns following 205 hours of sleep deprivation. Psy chosomatic Medicine, 1970, 32. 189-200.

MARON. L., RECHTSCHAFFEN, A., \& WOLPERT, E. A. Sleep cycle during napping. Archives of General Psychiatry, 1964, 11. 503-508. 
PhOEbUS, E., TAUB, J. M., Globus, G. G., \& DRURY, R. Sleep stage characteristics of extended sleep in normal subjects. Perceptual \& Motor Skills, $1970,31,526$.

RECHTSCHAFFEN, A., \& KALES, A. (Eds.) A manual of standardized terminology, techniques, and scoring system for sleep stages of human subjects. W a shington, D.C: U.S. Government Printing Office, 1968.

VERDONE, $P$. Sleep satiation: Extended sleep in normal subjects Electroencephalography \& Clinical Neurophysiology, 1968, 24, 417-423.

WEBB, W. B., \& AGNEW, H. W., JR. Sleep: Effects of a restricted regime. Science, $1965,150,1745-1747$

WEBB, W. B., \& AGNEW, H. W., JR. Sleep cycling within twenty-four hour periods. Journal of Experimental Psychology, 1967, 74, 158-160.

WEBB, W. B., AGNEW, H. W., JR., \& STERNTHAL, $H$. Sleep during the early morning. Psychonomic science, 1966, 6, 277-278.
WEITZMAN, E. D., KRIPKE, D. F., GOLDMACHER, D., McGREGOR, P., \& NOGEIRE, $C$. Acute reversal of the sleep-waking cycle in man: Effect on sleep stage patterns. Archives of Neurology, 1970, 22, 483-489.

WILLIAMS, H. L., HAMMACK, J. T., DALY, R. L., DEMENT, W. C., \& LUBIN, A. Responses to auditory stimulation, sleep loss and the EEG stages of sleep. Electroencephalography \& Clinical Neurophysiology, 1964, 16 , 269-279.

WILLIAMS, R. L., AGNEW, H. W., JR., \& WEBB, W. B. Sleep patterns in young adults: A n E G study Electroencephalography \& Clinical Neurophysiology, 1964, 17, 376-381.

WILLIAMS, R. L.. AGNEW, H. W.. JR., \& WEBB, W. B. Sleep patterns in the young adult female: An EEG study. Electroencephalography \& Clinical Neurophysiology, $1966,20,264-266$.

WINER, B. J. Statistical principles in experimental design. New York MeGraw-Hill, 1962 longer memory intervals showed greater accuracy on the test trials over all intervals. He suggested that Ss' information-processing strategies were a function of the demands of the task on which they were trained and that once a cognitive structure was learned in training, it would be transferred to the test trials. Group 2 Ss could perform adequately in their training trials by merely storing a few recency items in a short-term rehearsal buffer. However, Group 1 Ss had to employ a more permanent long-term code in training, which, when transferred to the test trials, presumably accounted for their superior performance at all intervals. Restle extrapolated from these findings the hypothesis that the phenomenon of improvement over trials in a memory task, commonly known as learning-to-learn or practice effects, might reflect the fact that it takes several trials for Ss to select the most efficient strategy for the task at hand rather than the refinement across trials of any one strategy.

The present experiment was designed to test Restle's hypothesis that Ss use the first few trials of a short-term memory task to select an appropriate acquisition strategy. The experiment uses a multiple-list, single-trial, single-probe serial memory task in which the Ss themselves determine the rate at which the lists' items are presented. Previous investigations using this task have provided Ss with from 5 to 10 "practice" trials to familiarize themselves with the task (Belmont \& Butterfield, 1969). Analyses of the Ss' interitem pauses have revealed that during the postpractice trials, Ss typically pause to rehearse near the middle of the lists but do not pause appreciably toward the end of the lists. The explanation for the use of this strategy is that recall of the probe from the early and middle portions of the lists requires that these items be transferred to a relatively permanent secondary memory store by means of rehearsal, which is accomplished during pauses near or just past the middle of the lists, whereas the terminal list items can be recalled accurately simply by committing them to an echo box or primary memory store.

If Ss adopt this sort of strategy gradually, then an examination of data from the first few trials, which are usually administered just to familiarize Ss with the task, should reveal evolving patterns of hesitations which gradually change to a stable pattern of pausing most just past the middle of the lists. SUBJECTS

The Ss were 11 undergraduate students who volunteered their services for $\$ 2.00$.
* The research reported here was supported by USPHS Grants HD-00183 and HD-04760. Reprint requests should be sent to the first author at 3933 Eaton Street, Kansas City, Kans. 66103 . pretrained two groups of Ss in us paired-associates task, group having relatively long presentation-to-test interval (Group 1) and the other experiencing shorter intervals (Group 2). Both groups were then tested on the same task with variable intervals. Restle found that the group trained with 